\title{
Fortalecimiento productivo a los criadores de cuy (Cavia porcellus) del trópico húmedo colombiano
}

\section{Productive strengthening of guinea pig breeders (Cavia porcellus) of the Colombian humid tropic}

\section{Fortalecimento produtivo de criadores de cobaias (Cavia porcellus) nos}

\section{trópicos úmidos colombianos}

\begin{abstract}
Ramírez Villa Luis Carlos ${ }^{1}$, Gómez Bilbao Pedro² y Murcia Rozo Jazmín ${ }^{3}$
${ }^{1}$ Licenciado en Producción Agropecuaria, (c)MSc., Docente Universidad de los

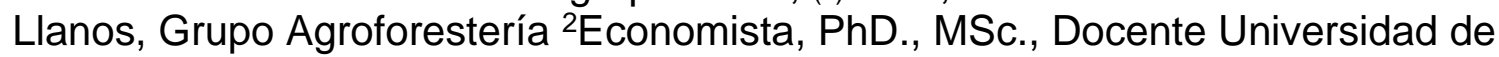
los Llanos, Grupo CEGER y ${ }^{3}$ Estudiante de MVZ, Grupo de Estudio Agroecología y Desarrollo Rural (ECODER)

Iramirez@unillanos.edu.co
\end{abstract}

Recibido 12 de Diciembre 2018, Aceptado 30 de Mayo 2019

\section{RESUMEN}

El proyecto se realizó en la región tropical húmeda, paisaje de la Orinoquia Colombiana, en Villavicencio, con el objetivo de generar un fortalecimiento productivo a los criadores de cuy (Cavia porcellus) a partir del diseño de un plan de acción para el mejoramiento y la asociatividad de los productores, se realizaron tres acciones puntales para ello, la primera un estudio socioeconómico de las familias que manifestaron su interés en la cría comercial de los animales lo que desencadenó en la segunda acción, puesto que con estos resultados se invitó a 10 familias para desarrollar un plan de capacitación sobre las buenas prácticas de producción del cuy en condiciones de la Orinoquia, capacitación que se ofreció por parte de la Universidad de los Llanos a través del grupo de estudio ECODER, utilizando el método constructivista de aprender haciendo, en el cual se desarrollaron estrategias de demostración de método, actividades de producción y el plan de negocio, para que las personas implementarán en sus fincas como unidades productivas, estas capacitaciones son el preámbulo para el desarrollo de la organización de los productores, ya que se sensibilizó a la comunidad participante 
para la creación de una asociación de productores de cuy en la Orinoquia, la cual será pionera en la producción de esta especie, debido a que en la región no existe. Los productores se han comprometido a través de la firma de una carta de intención para adelantar las gestiones correspondientes para el registro de la Asociación de Productores de Cuyes ante la Cámara de Comercio y poner en regla la reglamentación para operar desde un punto de vista económico y político la organización, el principal resultado que se ha logrado dentro del proyecto es la divulgación de la producción de la carne de cuy, un aumento de la producción de cuy y la organización de los productores para emprender procesos colectivos que garantice la seguridad alimentaria y el flujo de caja familiar.

Palabras clave: Cobayo, productividad, organización comunitaria, desarrollo rural.

\begin{abstract}
The project was carried out in the humid tropical region, landscape of the Colombian Orinoquia, in Villavicencio, with the aim of generating a productive strengthening of guinea pig breeders (Cavia porcellus) from the design of an action plan for the improvement and associativity of producers, three main actions were carried out for this, the first a socioeconomic study of the families that expressed their interest in the commercial rearing of the animals which triggered in the second action, because with these results, 10 families were invited to develop a training plan on the good production practices of the guinea pig under Orinoquia conditions, training offered by the University of Los Llanos through the ECODER study group, using the constructivist method of learning by doing, in which method demonstration strategies, production activities and the business plan were developed, So that people will implement in their farms as productive units, these trainings are the preamble for the development of the organization of the producers, since the participating community was sensitized for the creation of an association of guinea pig producers in the Orinoquia, the which will be a pioneer in the production of this species, because it does not exist in the region. The producers have committed themselves by signing a letter of intent to advance the corresponding steps for the registration of the Association of Guinea Pig Producers before the Chamber of
\end{abstract}


Commerce and to regulate the regulations to operate from an economic point of view and political organization, The main result that has been achieved within the project is the dissemination of guinea pig production, an increase in guinea pig production and the organization of producers to undertake collective processes that guarantee food security and family cash flow.

Keywords: Guinea pig, productivity, community organization, rural development.

\section{RESUMO}

O projeto foi realizado na região tropical úmida, paisagem da Orinoquia colombiana, em Villavicencio, com o objetivo de gerar um fortalecimento produtivo dos criadores de cobaias (Cavia porcellus) a partir da elaboração de um plano de ação para a melhoria e a associatividade dos produtores, foram realizadas três ações principais para isso, a primeira um estudo socioeconômico das famílias que manifestaram interesse na criação comercial dos animais, desencadeada na segunda ação, sendo que com esses resultados, 10 famílias foram convidadas a desenvolver um plano de treinamento sobre as boas práticas de produção do porquinho-da-índia nas condições de Orinoquia, treinamento oferecido pela Universidade de Los Llanos por meio do grupo de estudo ECODER, usando o método construtivista de aprender fazendo, no qual estratégias de demonstração de método, atividades de produção e plano de negócios foram desenvolvidos, Para que as pessoas implementem em suas fazendas como unidades produtivas, esses treinamentos são um preâmbulo para o desenvolvimento da organização de produtores, uma vez que a comunidade participante foi sensibilizada pela criação de uma associação de produtores de porquinhos-da-índia no Orinoquia, o que será pioneira na produção dessa espécie, porque ela não existe na região. Os produtores se comprometeram assinando uma carta de intenções para avançar as etapas correspondentes para o registro da Associação de Produtores de Cobaias na Câmara de Comércio e regulamentar os regulamentos para operar do ponto de vista econômico e organização política, principal resultado alcançado no projeto é a disseminação da produção de cobaias, o aumento da produção de cobaias e a organização dos produtores para realizar 
processos coletivos que garantam a segurança alimentar e o fluxo de caixa da família.

Palavras-chave: Porquinho da índia, produtividade, organização comunitária, desenvolvimento rural.

\section{INTRODUCCIÓN}

En la Orinoquia Colombia se encuentran las condiciones ecofisiográficas básicas para que la producción de Cavia porcellus sea un éxito desde el punto de vista biológico y su rendimiento productivo se pueda expresar claramente, sin embargo, la comercialización de la carne en pie y en canal es un problema a la hora de pensar en un sistema de producción intensivo de cuyes, ello debido a la cultura de los consumidores y poca publicidad que tiene el consumo de carne de cuy en los Llanos.

Esta limitante es preocupante debido a que es una especie de fácil manejo y buen comportamiento biológico para la reproducción y que se puede adaptar a las condiciones de la Orinoquia lo que representa un rezago regional en cuanto a que no se han estandarizado los procesos de producción y comercialización, sin embargo, se busca demostrar que la cría del cuy en un proceso bien administrado puede generar un buen flujo de caja para las familias rurales y reforzar la seguridad alimentaria produciendo una fuente proteína para completar adecuadamente los requerimientos nutricionales de la familia (Xicohtencatl, 2013).

Como ya ha sido señalado, el mercado de la carne de cuy en la Orinoquia no se ha explorado seriamente, debido a la falta de oferta, sin embargo, la demanda existe porque algunos productores, desconociendo la realidad deciden de manera individual comerciar los animales en pie, muchas veces no teniendo en cuenta las posibilidades técnicas y financieras que pueden hacer posible tratos justos en la comercialización de la carne en pie o en canal, por tal motivo la pregunta a abordar en el presente proyecto es: ¿Qué acciones se pueden proponer para el mejoramiento del sistema de producción y comercialización de cuy a pequeños productores, como alternativa de la agricultura familiar en la Orinoquía? 
Para atender la problemática se planteó un trabajo mancomunado con los productores agropecuarios de la región, el cual consistió en determinar las potencialidades a través de un diagnostico socioeconómico y productivo, después de esto se diseñó un plan de acción para el mejoramiento de la calidad de vida de las familias dedicadas a la actividad cuyicula en la Orinoquía, en el cual se establecieron las acciones de capacitación, por último se identificaron las estrategias para su organización, buscando una alternativa para el desarrollo rural, aplicando el principio de acción participativa de Balcázar, (2003).

Este estudio se llevó a cabo en el piedemonte llanero el cual se encuentra ubicado en el departamento del Meta entre los 700 y $500 \mathrm{msnm}$, con temperaturas medias de 23 a $30 \stackrel{\circ}{\circ}$ y un régimen de lluvias bi-estacional con 3000 a $4000 \mathrm{~mm}$ de precipitación anual. El piedemonte llanero cuenta con aproximadamente 200.000 hectáreas destinadas para agricultura comercial y ganadería intensiva y 453.775 hectáreas destinadas a ganadería extensiva, reforestación o conservación de la vegetación natural (Botero, 1999).

La producción de cuyes ha aumentado en la región de la Orinoquia, ello se debe en gran parte a que desde el año 2013 se constituyó la unidad de producción de cuyes de la Universidad de los Llanos, donde actualmente existen 10 núcleos en diferentes comunidades en las que se ha impulsado el aprovechamiento de esta especie para generar una proteína de origen animal económica y fácil de manejar, en el proceso se pueden utilizar recursos locales en cuanto a su establecimiento y nutrición, a partir de forrajes propios de la región, lo que enriquece su valor para la seguridad alimentaria en comunidades rurales en condición de vulnerabilidad. La inversión inicial para un proyecto de producción comercial con esta especie no es alta, todo lo contrario, la principal problemática identificada por el grupo de estudio en ECODER de la Facultad de Ciencias Agropecuarias y Recursos Naturales de la Universidad de los Llanos es la comercialización.

Es así que se requiere una cadena de comercialización segura para poder recuperar, con tranquilidad, la inversión de los productores y garantizar un flujo de caja constante que generé una buena relación costo beneficio, lo que redundaría en 
mejorar la calidad de vida para la familia productora, sin embargo el proceso de comercialización requiere varios eslabones de una cadena, el primero de ellos el productor, que debe garantizar volúmenes de producción para poder asumir una verdadera participación en el mercado, de igual manera se requiere establecer estrategias para la implementación, el manejo, el faenado y la venta, por lo cual es necesario el fortalecimiento organizativo de los productores de cuyes en la Orinoquia Colombiana.

\section{METODOLOGÍA}

El proceso se realizó a partir de un diseño metodológico no experimental, descriptivo y transversal (Hernández et al., 2010), para lo cual se recurrió a fuentes de información primaria utilizando variables independientes útiles para contextualizar la zona de estudio. Para ello se realizó una encuesta a los propietarios de unidades productivas que implicó visitas a las diferentes fincas para obtener información de los sistemas encontrados por medio de cuestionarios que se elaboraron para ser tabulados en una estadística descriptiva no paramétrica, una vez obtenida esta información se realizó un plan de capacitación con 10 familias seleccionadas, para estas capacitaciones se elaboró una serie de módulos didácticos con la metodología aprender haciendo desde un enfoque constructivista.

El estudio se realizó en diferentes veredas rurales del departamento del Meta las cuales abarcaron los municipios de Villavicencio, Puerto López, Cumaral y San Martin. Dentro del municipio de Villavicencio se encuestaron un total de 24 fincas productivas que comprendieron el corregimiento 1 con las veredas La cumbre y San Luis de Ocoa; corregimiento 2 con las veredas de Buenavista, Servitá, Samaria, Contadero; corregimiento 3 con las veredas Palmichal y la Argentina; corregimiento 4 con la vereda Alto Pompeya y Caños negros, finalmente el corregimiento 6 con las veredas de Lourdes, San José Bajo y Santa Helena Alta.

Se realizó un muestreo discrecional o por juicio debido a que el estudio socioeconómico es susceptible a todos los sistemas de producción a todos los productores del piedemonte llanero, sin embargo, se tuvo en cuenta solamente los 
que se encuentran registrados en la base de datos de la UMATA de Villavicencio y que manifestaron su interés por la producción de cuyes.

Las fuentes de información primaria a las que se recurrió son las unidades de análisis, es decir las familias de las fincas cuya actividad principal sea agropecuaria. A estas familias previamente seleccionadas por la base de datos de la UMATA de Villavicencio se les realizó una visita con el fin de aplicarles la encuesta y tomar algunos datos de observación directa.

En el municipio de Puerto López se encuestó una finca ubicada en la vereda Alto Menegua; en las veredas Cruce de Guacavía, San Nicolás y Caney medio se encuestaron con tres fincas del municipio de Cumaral. El municipio de San Martin vereda el Merey y El calvario vereda San Francisco se encuestó una finca en cada uno de los municipios para un total de 30 productores que aparecen en la Tabla 1 , de los cuales unos hacen parte de la base de datos de la UMATA y otros no, pero que mostraron interés en la producción de Cavia porcellus.

Con ayuda de la base de datos de la UMATA se realizaron llamadas telefónicas con la finalidad de invitar a los pequeños productores interesados en iniciar capacitaciones para poder establecer el sistema productivo de Cavia porcellus. En este primer encuentro se les dio a conocer a los productores los objetivos del proyecto y se estableció un cronograma de las capacitaciones a realizar en el marco de un curso taller sobre las Buenas Prácticas de Producción de Cuy (Cavia porcellus).

Como fuente de información secundaria se tomó la cartografía del IGAC, (2015), la cual fue crucial para ubicar geográficamente las fincas productivas, además de ser una fuente importante de información social y geográfica de las zonas, además de se comparó el uso del suelo con el plan de ordenamiento territorial (POT) aprobado para Villavicencio.

En los métodos de recolección de la información se consideraron variables técnicas, económicas y sociales pertinentes para caracterizar y describir los sistemas productivos bajo el estudio y se realizó el análisis socioeconómico. Para ello se hizo 
uso de entrevistas, encuestas, conversaciones informales, observación en campo y consultas personales (Forero et al, 2015). Para el análisis socioeconómico de los sistemas productivos en el estudio se utilizó estadística descriptiva recurriendo a las medidas de tendencia central (Hernández et al., 2010).

En cuanto al análisis social se abordó desde la demanda de jornales, la generación de empleo y la seguridad alimentaria. Para complementar se realizó un análisis cuantitativo comparativo de los sistemas con la finalidad de evaluarlos. Para la ejecución de la metodología se elaboró un cuestionario donde se tuvieron en cuenta variables independientes útiles para contextualizar la zona de estudio. Este cuestionario se aplicó a cada uno de los propietarios de las fincas que mostraron interés en recibir capacitación para emprender la producción de cuyes.

\section{RESULTADOS}

Se realizaron 30 encuestas en las que se referenció la Unidad Agrícola Familiar a cada uno de los productores (Tabla 1), este instrumento contó con elementos fundamentales para un diagnostico socioeconómico, tal como grado de escolaridad, número de miembros del hogar, el trabajo desempeñado por cada uno de ellos, acceso a condiciones básicas de salud y educación, por otro lado, se realizaron preguntas sobre seguridad alimentaria, la frecuencia en el consumo de proteína de origen animal y la disponibilidad de la crianza de animales alternativos a la dieta cultural que se tiene para la región.

Se puede mencionar que el $80 \%$ de las fincas que participaron en este estudio socioeconómico están ubicadas en Villavicencio, seguido de un $10 \%$ en Cumaral y finalmente un $3.3 \%$ que corresponde de manera individual a los municipios del Calvario, Puerto López y San Martin. Del total de productores junto con su núcleo familiar abarcan 115 personas, y 95 de estas cuentan con SISBEN y 20 no lo tienen, lo que equivale a un $82.60 \%$ y $17.39 \%$ de la población respectivamente. Del $82.60 \%$ que tienen SISBEN, el $95.78 \%$ que corresponde a 91 personas cuentan con un nivel 01 en SISBEN y tan solo un $4.21 \%$ tienen un nivel 02 . Referente al sistema de salud de la población rural sometida a estudio el $84.34 \%$ cuentan con un régimen de salud 
subsidiado, un $10.34 \%$ tienen un régimen de salud contributivo y tan solo un $5.21 \%$, su sistema de salud es especializado.

Tabla 1. Unidades agrícolas familiares encuestadas y su ubicación

\begin{tabular}{|c|c|c|c|}
\hline № & Nombre Finca & Vereda & Municipio \\
\hline 1 & Las Arenas & & \\
\hline 2 & Villa Zulay & & \\
\hline 3 & Villa Laura & Cañoc Nearnc & \\
\hline 4 & Villa Luz & & \\
\hline 5 & Los mandarinos & & \\
\hline 6 & Quinta El Paraíso & La Argentina & \\
\hline 7 & las Esperanza & San Luis de Ocoa & \\
\hline 8 & Agua Bonita & Palmichal & \\
\hline 9 & La Humana & La Floresta & \\
\hline 10 & Los naranjos & Santa Helena & \\
\hline 11 & San Miguel Arcángel & Lourdes & \\
\hline 12 & Los Corrales & & Villaviconcin \\
\hline 13 & El refugio & & viliavicenticio \\
\hline 14 & Santa Teresa & Samaria & \\
\hline 15 & Fantasía de la Montaña & & \\
\hline 16 & La Florida & & \\
\hline 17 & El Vergel & San José Bajo & \\
\hline 18 & La Fortuna & Contadero & \\
\hline 19 & La Palmita & & \\
\hline 20 & El Cascal & Servità & \\
\hline 21 & San Matías & & \\
\hline 22 & Villa Berraca & La Cumbre & \\
\hline 23 & Miraflores & Santa Helena & \\
\hline 24 & El Zafiro & Bajo Pompeya & \\
\hline 25 & San Antonio & Cruce De Guacavia & \\
\hline 26 & La Floresta & Inspección San Nicolás & Cumaral \\
\hline 27 & san Juanito & Caney Medio & \\
\hline 28 & El porvenir & San Francisco & El calvario \\
\hline 29 & la fortuna & El Merey & San Martin \\
\hline 30 & el mirador & Alto Menegua & Puerto López \\
\hline
\end{tabular}


Con relación a la alimentación familiar, el 100\% de la población consume carne, se obtuvo que 12 familias la consumen diariamente, 12 familias cada tercer día y 6 familias la consumen semanalmente, lo que corresponde a un $40 \%, 40 \%$ y $20 \%$, respectivamente. Lo que indica que un $40 \%$ de la población rural del Pie de Monte estaría en un riesgo de seguridad alimentaria, mientras un $20 \%$ ya se encuentra en peligro de desnutrición, y que solo un $40 \%$ no presentaría riesgo (Gráfica 1). Con respecto a cómo adquieren la carne para su consumo sea diario, cada tercer día o semanalmente se encontró que de las 30 familias en su mayoría la adquieren del supermercado abarcando un $66.66 \%$ seguido de un $40 \%$ que la adquieren en su propia finca lo cual corresponde a un total de 12 familias, un $10 \%$ compran la carne para su consumo en la central de abastos, 3 familias optan por comprarla en la fama o en la vereda con un $6.66 \%$ y $3.33 \%$ respectivamente.

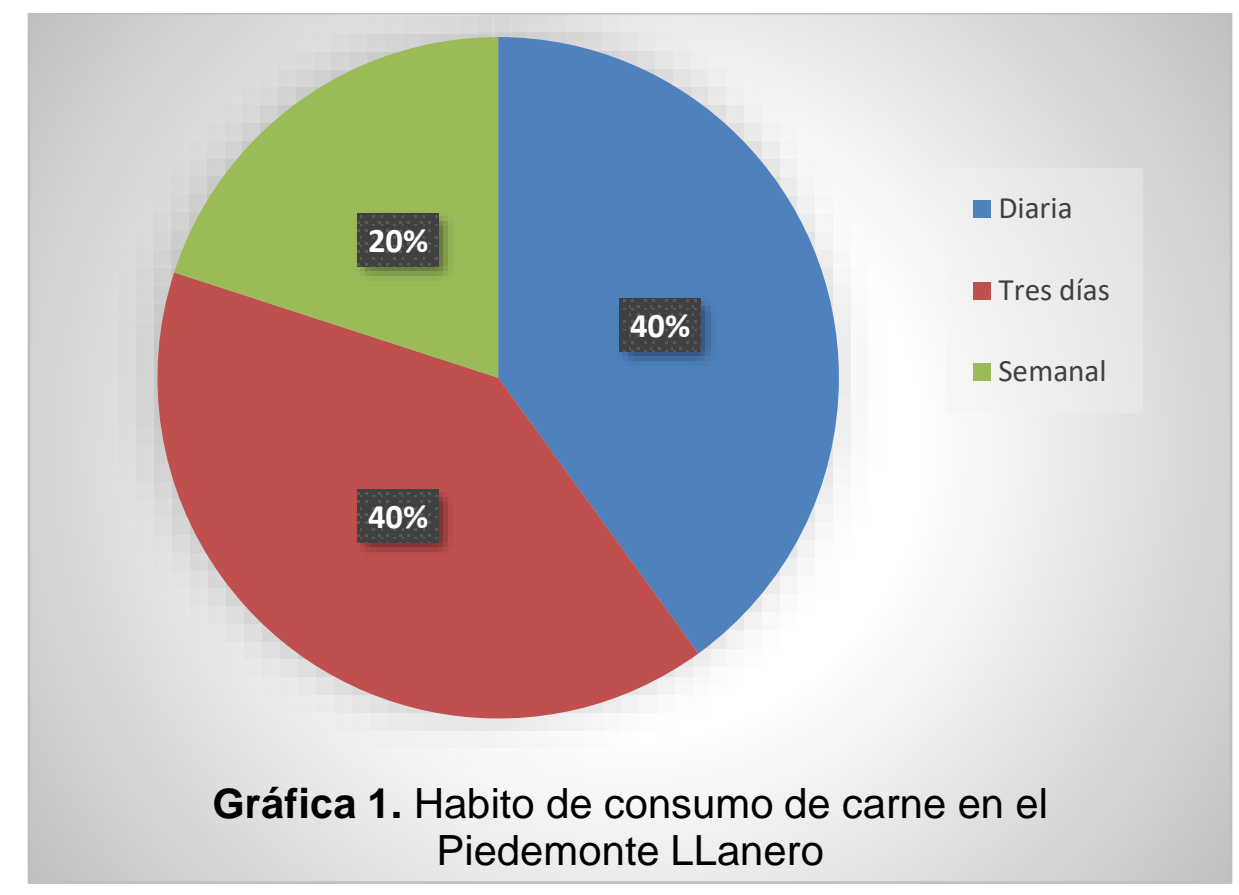

Frente a la frecuencia de los productos consumidos, que aportan proteína de origen animal, se puede deducir que el consumo de huevo es alto, en promedio 29 unidades semanales, analizando los diversos productos que utilizan las familias campesinas del piedemonte llanero, que son en promedio de cuatro integrantes, le sigue el consumo de huevo y leche, mientras que el consumo de productos no 
convencionales culturalmente como lo es el kumis con menos de una unidad de medida (Tabla 2).

Tabla 2. Consumo promedio de proteína de origen animal por familias rurales de cuatro integrantes en el pie de monte llanero

\begin{tabular}{cccc}
\hline Producto & $\begin{array}{c}\text { Consumo } \\
\text { Semanal }(\mathrm{lb})\end{array}$ & Producto & $\begin{array}{c}\text { Consumo } \\
\text { Semanal }\end{array}$ \\
\hline Bovino & 3.23 & Leche $(\mathrm{L})$ & 4.21 \\
Ovino & 0.03 & Huevos (unidad) & 28.33 \\
Porcino & 1.9 & Queso $(\mathrm{lb})$ & 1.6 \\
Caprino & 0 & Mantequilla $(\mathrm{lb})$ & 0.26 \\
Pez & 1.9 & Yogurt $(\mathrm{L})$ & 0.55 \\
Pollo & 4.46 & Kumis $(\mathrm{L})$ & 0.05 \\
\hline
\end{tabular}

En cuando al consumo de especies silvestres se encontró que, si se hace pero con muy baja frecuencia; dentro de estas la especie que más se consume es la lapa con un promedio de 12.8 libras seguido del gürre con $1.8 \%$ de consumo. En menor proporción, los productores también comen como fuente de proteína: babilla, cerdo de monte, chigüiro, conejo y cuy en promedio de 4, 4, 3, 1.5 y 1.5 libras respectivamente.

Al momento de realizar las encuestas a los productores estos manifestaron el interés para la producción de Cavia porcellus debido a que esta especie requiere de poco espacio, es de fácil manejo, a su vez de que en sus fincas cuentan con gran variedad de especies forrajeras aptas para la alimentación de los cuyes, la finalidad de un gran porcentaje de estos productores es para consumo propio y comercialización de la especie.

Dado el gran porcentaje de familias rurales que tienen interés de iniciar la producción de Cavia porcellus en Villavicencio, se hizo uso de la cartografía del IGAC, (2015) para ubicar geográficamente las veredas donde se encuentran las fincas de los productores que mostraron el interés en asistir a las capacitaciones (Gráfica 2). 


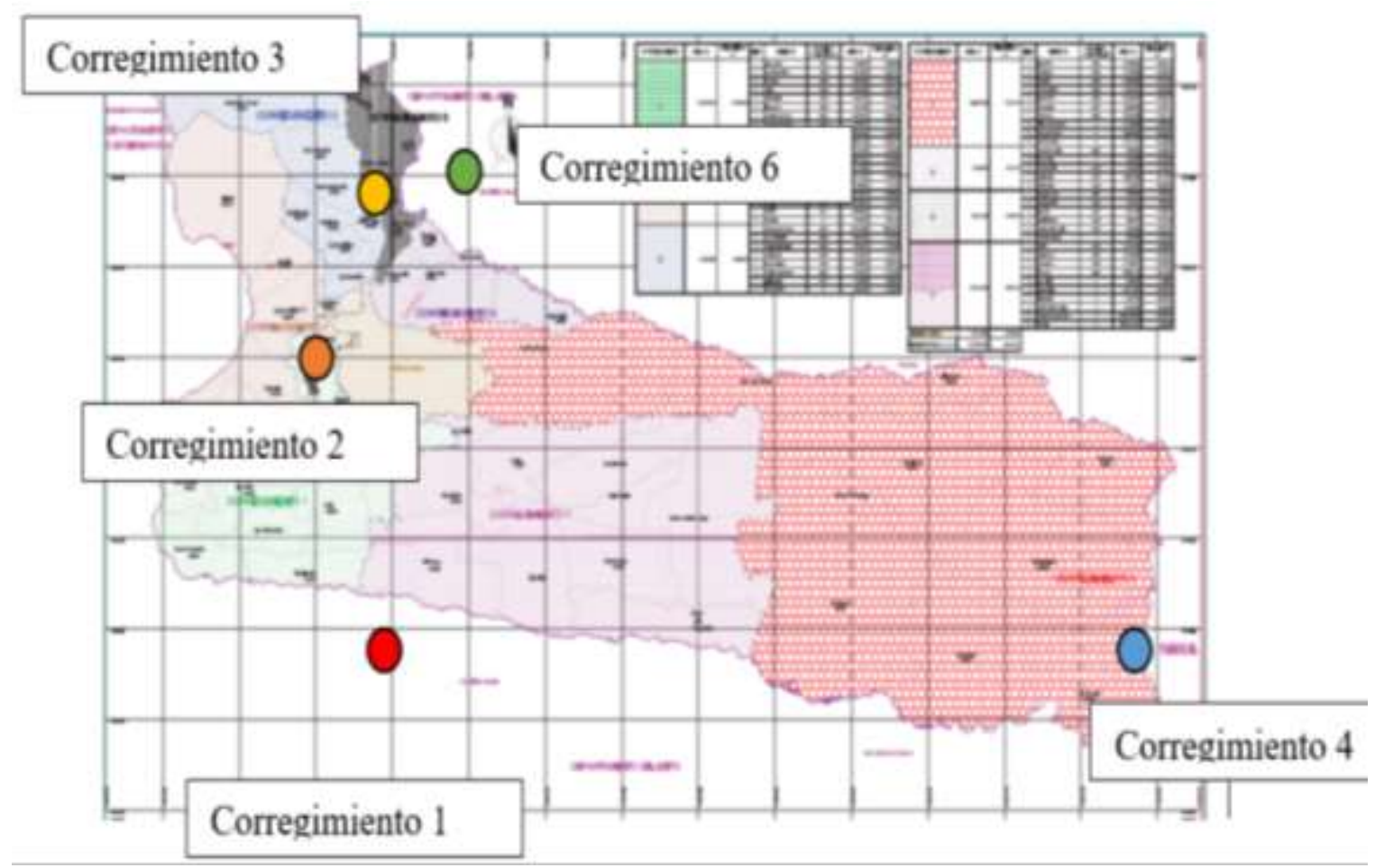

Gráfica 2. Veredas de Villavicencio donde los productores están interesados en la producción de cuyes

La segunda fase del proceso se desarrolló a partir de las capacitaciones, las cuales se realizaron teniendo en cuenta la metodología aprender haciendo desde un enfoque constructivista, siendo los facilitadores estudiantes de Medicina Veterinaria y Zootecnia y algunos docentes del área técnica de la producción de gestión empresarial rural, para ello se utilizaron los postulados del aprendizaje colectivo, en las cuales se centra el aprendizaje en la generación de interés (Maldonado, 2008).

Se diseñó un curso de buenas prácticas de producción de cuy en el contexto de la Orinoquia Colombiana, en el cual se abordaron temáticas definidas como aspectos generales de la producción de cuy, instalaciones y equipos, manejo del sistema productivo, nutrición, sanidad, transformación de canales, comercialización y organización comunitaria, para un total de 48 horas, 24 presenciales y 24 de tiempo independiente; para el aprendizaje del manejo técnico del cuy se tomó como referencia el manual de Vivas y Carballo, (2013) (Figuras 1 y 2). 


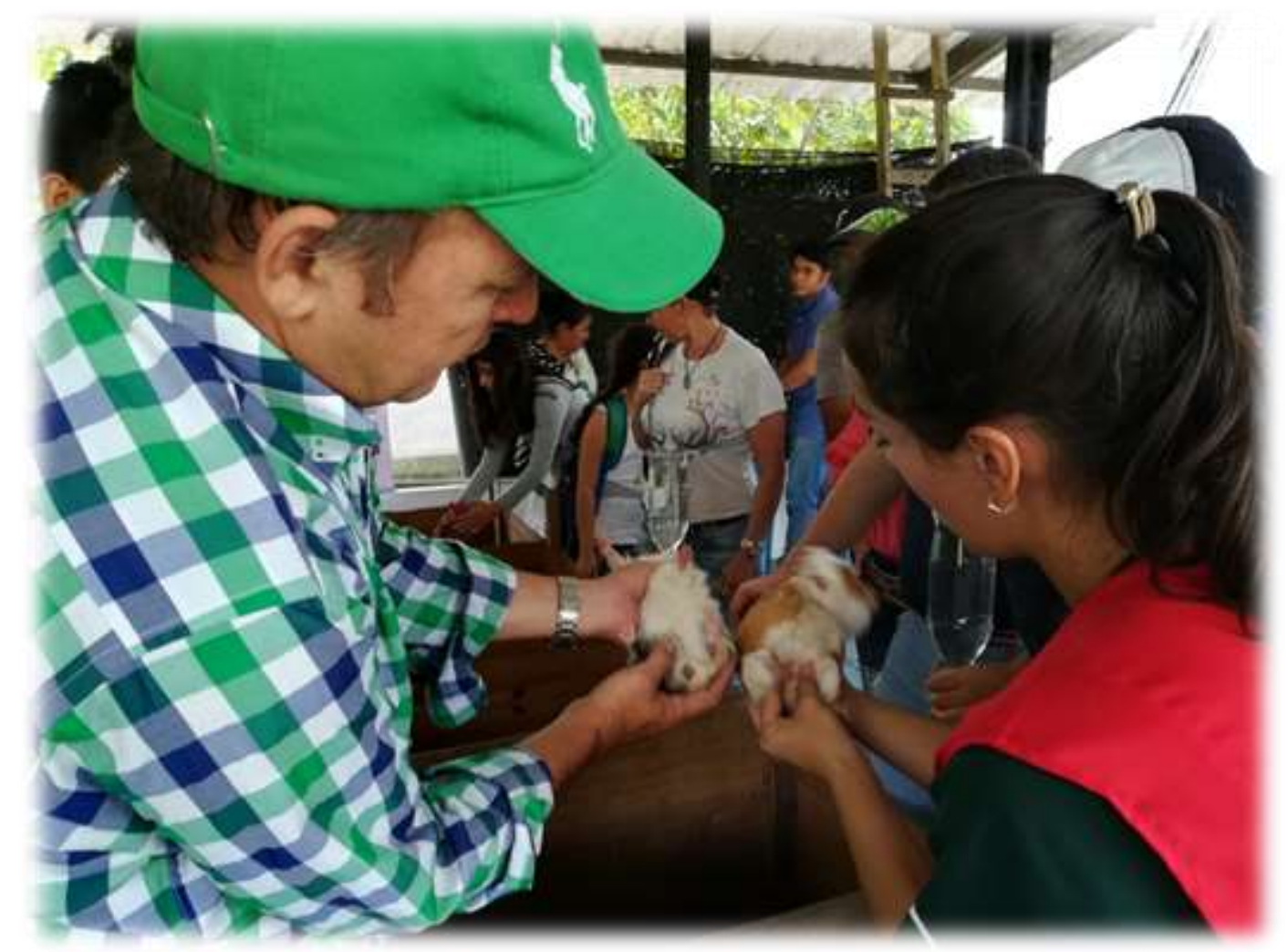

Figura 1. Los docentes de la Universidad explicaron la practicas para el manejo de los cuyes a los productores. Fuente: Autores.

La participación activa en la capacitación por parte de los productores genera las condiciones adecuadas en la región sobre el de conocimiento del manejo de la especie para que se implemente la producción de Cavia porcellus de forma comercial, además que se espera que el dialogo entre productores desencadene una forma de asociatividad que comprenda desde la adquisición de líneas genéticas, la generación de dietas con forraje de la zona y la nuevas formas de mercadotecnia para la comercialización del producto final.

\section{ANÁLISIS}

El presente proyecto ha desarrollado tres impactos fundamentales en el trabajo comunitario, el primero desde el componente social, el segundo desde el componente económico y el tercero desde el componente técnico, estos sin mencionar el carácter pedagógico. 


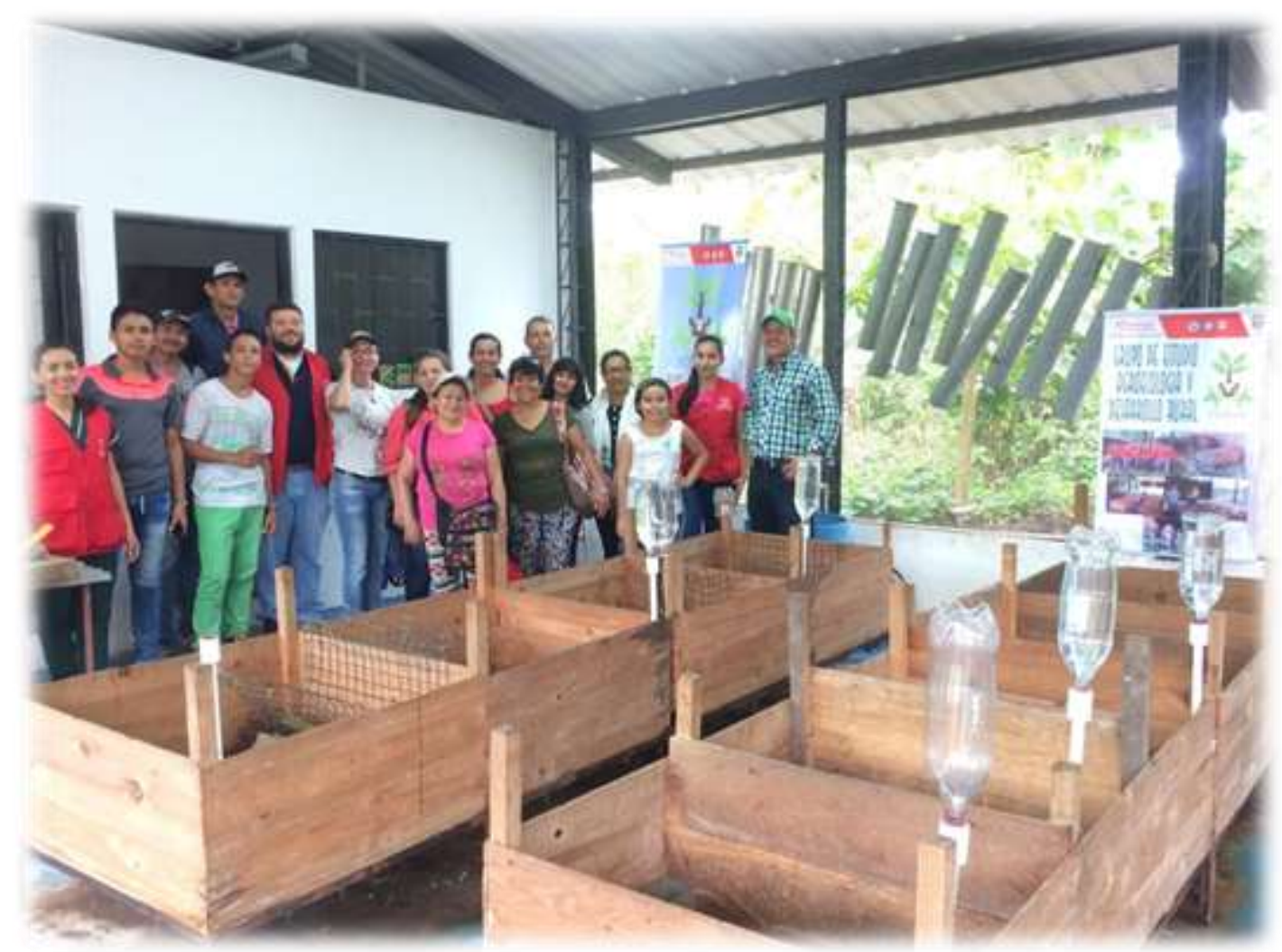

Figura 2. Las instalaciones son un factor importante para la producción del cuy. Fuente: Autores.

El impacto social ha sido prioritario para el desarrollo del proyecto, este tiene dos dimensiones, la primera la capacidad de ocupación de mano de obra familiar, para lo cual, con la caracterización de la comunidad y el desarrollo de las capacitaciones, se buscó que se diera una integración adecuada de las familias involucradas en el proyecto, el número de familias involucradas en el proceso fue 30 , de esta se seleccionaron 10, a las cuales se les ha realizado un seguimiento estableciendo una metodología de trabajo, esto con el fin de involucrar en la crianza del cuy a toda la familia.

El desarrollo de la actividad económica es el proceso central del presente proyecto, el cuy es una posibilidad económica para los pequeños productores de la región, se aumenta la producción en términos de cada familia porque se iniciará con un pie de cría definido en la demostración de método desarrollada por la Universidad, esta estrategia se establece para 10 núcleos productivos a las familias rurales. 
Al generar la asociación de productores de cuyes en la Orinoquia aumentan los niveles de participación y la visibilidad de estos en cuanto a las políticas de producción, el mercado y la economía en general.

\section{CONCLUSIONES Y RECOMENDACIONES}

La producción de cuy (Cavia porcellus) es una alternativa para la seguridad alimentaria, proporcionaría una solución rápida y de fácil manejo para la población rural que le proveería proteína de origen animal, lo que enriquecerá su dieta.

En cuanto a su comercialización la producción de Cavia porcellus puede ser una alternativa para generar flujo de caja en las familias rurales que desarrollen la cría del cuy de forma comercial.

Desde el punto de vista social, la comunicación personal con cada productor es esencial ya que cada uno va argumentando su proyecto, algunos desean la producción para comercialización, otros como seguridad alimentaria o simplemente por gusto. Es importante visitar cada finca con el fin de ver que con que instalaciones se cuenta para a si mismo dar ideas de un diseño artesanal que sea económicamente viable para iniciar la producción y así mismo la asesoría para la nutrición, esto con el fin de aprovechar la biodisponibilidad de forrajes se cuenta en la zona.

\section{REFERENCIAS BIBLIOGRÁFICAS}

1. Balcázar, F. Investigación acción participativa (IAP): Aspectos conceptuales y dificultades de implementación. Fundamentos en humanidades, 4 (7-8): 59-77. 2003.

2. Botero, P. (Ed). Paisajes fisiográficos de Orinoquia- Amazonia (ORAM) Colombia. Análisis geográficos N. 27-28, Bogotá, Colombia: Instituto Geográfico Agustín Codazzi (IGAC). 361 p. 1999.

3. Xicohtencatl, P., Barrera, S., Orozco, T., Torres, S., Monsivais, R. Parámetros productivos de cuyes (Cavia porcellus) del nacimiento al sacrificio en Nayarit, México. Abanico Veterinario, 3 (1): 36-43. 2013.

4. Forero, J., Yunda, C., De Vargas, M., Rodríguez, C., León, A. La viabilidad de la agricultura familiar en la altillanura colombiana. Análisis de su eficiencia económica - productiva y su dinámica ecosistémica en comunidades de Puerto López. Bogotá, Colombia: Espacio Creativo Impresores SAS. 63 p. 2015. 
5. Heredia, A., Vargas, J. Alimentación con morera (Morus spp.), maralfalfa morado (Pennisetum spp.) y mezcla forrajera en cuyes (Cavia porcellus) de 15 días hasta los 3 meses de edad en el criadero del CEYPSA. Tesis de Médico Veterinario y Zootecnista. Unidad Académica de Ciencias Agropecuarias y Recursos Naturales, Universidad Técnica de Cotopaxi, Latacunga, Ecuador 97 p. 2010.

6. Instituto Geográfico Agustín Codazzi (IGAC). Plano de sistema de soporte ambiental suelo rural. $2015 . \quad$ Disponible En: http://antigua.villavicencio.gov.co/index.php?option=com docman\&task=\&gi $\mathrm{d}=1638 \&$ ltemid $=136$.

7. Instituto Geográfico Agustín Codazzi (IGAC). Informe de gestión 2008. Bogotá, Colombia: Imprenta Nacional de Colombia. 162 p. 2008. Disponible En: http://www2.igac.gov.co/igac web/UserFiles/File/GESTION INSTITUCION AL 2009/Informe Gestion 2008 IGAC.pdf.

8. Maldonado, M. Aprendizaje basado en proyectos colaborativos. Una experiencia en educación superior. LAURUS Revista de Educación, 14 (28): 158-180. 2008.

9. Meza, G., Cabrera, R., Morán, J., Meza, F, Cabrera, C., Meza, C., Meza, J., Cabanilla, M., López, F., Pincay, J., Bohórquez, T., Ortiz, J. Mejora de engorde de cuyes (Cavia porcellus L.) a base de gramíneas y forrajeras arbustivas tropicales en la zona de Quevedo, Revista IDESIA, 32 (3): 75-80.

10. Hernández, R., Fernández, C., y Baptista, M. 2010. Metodología de la investigación. 5a Ed. México: Ed McGraw-Hill. 613 p. 2014.

11. Salcedo, S. Guzmán, L. (Eds.). Agricultura familiar en América Latina y el Caribe. Recomendaciones de política. Santiago de Chile: Organización de las Naciones Unidas para la Alimentación y la Agricultura (FAO). 497 p. 2014. Disponible En: http://www.fao.org/3/i3788S/i3788S.pdf.

12. Vivas, J., Carballo, D. Especies alternativas: Manual de crianza de cobayos (Cavia porcellus). $1^{\underline{a}}$ Ed. Managua, Nicaragua: Universidad Nacional Agraria. 81 p. 2013. Disponible En: http://cenida.una.edu.ni/textos/nl01v856e.pdf 